\title{
Localized gastrointestinal stromal tumor of the rectum: An uncommon primary site with prominent disease and treatment-related morbidities
}

\author{
MOHAMAD FARID $^{1 *}$, MARCUS JIN FU LEE ${ }^{5 *}$, MIN HOE CHEW ${ }^{2}$, WHEE SZE ONG ${ }^{3}$, \\ ALISA NOOR HIDAYAH SAIRI ${ }^{1}$, KIAN FONG FOO ${ }^{4}$, SU PIN CHOO ${ }^{1}$, WEN HSIN KOO ${ }^{1}$, \\ SIMON ONG ${ }^{1}$, POH KOON KOH ${ }^{2}$ and RICHARD QUEK ${ }^{1}$
}

\author{
${ }^{1}$ Department of Medical Oncology, National Cancer Centre Singapore, $169610 ;{ }^{2}$ Department of Colorectal Surgery, \\ Singapore General Hospital, 169608; ${ }^{3}$ Department of Clinical Trials and Epidemiological Sciences, \\ National Cancer Centre Singapore, 169610; ${ }^{4}$ Parkway Cancer Centre, Gleneagles Hospital, 258500; \\ ${ }^{5}$ Yong Loo Lin School of Medicine, National University of Singapore, 119228, Republic of Singapore
}

Received July 10, 2012; Accepted September 13, 2012

DOI: $10.3892 / \mathrm{mco} .2012 .25$

\begin{abstract}
Well-established clinicopathological variables used in the risk stratification of gastrointestinal stromal tumor (GIST) may not completely predict rectal GIST, an uncommon and poorly studied GIST subset. The aim of the present study was to determine the patterns of relapse and morbidities associated with recurrence in rectal GIST. A single-institution retrospective study between 2002 and 2011 was conducted, identifying 9 patients $(8 \%)$ with localized rectal GIST, while comparing small intestinal $(n=37)$ and gastric ( $n=63$ ) GIST (median age, 60 years). Rectal GIST tumors were smaller compared to small intestinal/gastric GIST $(\mathrm{P}=0.044)$. The number of mitoses per 50 high-power field (HPF) did not differ by primary site. In general, $73 \%$ of patients were high-risk, as defined by the National Institutes of Health (NIH) consensus criteria, however, only $25 \%$ received adjuvant imatinib. Fewer rectal GIST patients achieved negative surgical margins compared to small intestinal/gastric GIST (67 vs. 92\%; P=0.054). Of the 9 patients with localized rectal GIST 6 had peri-operative tumor rupture, anastomotic breakdown or required anal sphincter-compromising surgery. At the time of the first relapse, $83 \%$ of the recurrences were local failures for rectal GIST, compared to $21 \%$ for small intestinal/gastric GIST $(\mathrm{P}=0.005)$. The median relapse-free
\end{abstract}

Correspondence to: Dr Richard Quek, Department of Medical Oncology, National Cancer Centre Singapore, 11 Hospital Drive, Singapore 169610, Republic of Singapore

E-mail: dmorqhh@nccs.com.sg

"Contributed equally

Key words: gastrointestinal stromal tumor, rectal gastrointestinal stromal tumor, localized gastrointestinal stromal tumor, local morbidity, local relapse survival was 51 months for the entire cohort, and 54, 36 and 56 months for rectal, small intestinal and gastric GIST, respectively $(\mathrm{P}=0.468)$. Rectal GIST was found to be associated with high rates of local relapse and significant morbidity, despite being significantly smaller compared to GIST of other sites. A multimodality peri-operative therapeutic approach may be required to improve outcomes.

\section{Introduction}

Over the past decade, significant advancements have been made in the prognosis and management of resected gastrointestinal stromal tumor (GIST). Risk stratification models have been developed and are used in routine clinical practice to provide a prognosis for patients with localized GIST. These models serve to guide the use of adjuvant imatinib therapy following curative surgery. The National Institutes of Health (NIH) consensus criteria used tumor size and number of mitoses per 50 high-power field (HPF) to stratify patients into four risk cohorts (1). Miettinen and Lasota (2) retrospectively reviewed data from the Armed Forces Institute of Pathology, comprising the largest reported series of more than 2000 cases of GIST, to assign specific risks of relapse based upon size, number of mitoses and anatomic location. In their study, Joensuu and colleagues (3) suggested an update of the NIH consensus criteria incorporating anatomic location and presence of tumor rupture occurring spontaneously or at the time of surgery, following the establishment of tumor rupture as an independent adverse risk factor for disease relapse (4).

Imatinib, a small molecule tyrosine kinase inhibitor of the KIT oncoprotein, is of proven benefit in the adjuvant treatment of patients with completely resected GIST. In the randomized phase III ACOSOG Z9001 study evaluating patients with GIST of at least $3 \mathrm{~cm}, 1$ year of adjuvant imatinib improved recurrence-free survival (5). More recently, the SSGXVIII study enrolled 400 high-risk GIST patients based on the modified NIH consensus criteria (3) and randomized the patients into 3 years vs. 1 year of adjuvant imatinib. Preliminary results 
demonstrated that 3 years of adjuvant imatinib significantly improved 5-year recurrence-free survival (65.6 vs. 47.9\%) and overall survival (OS) (92.0 vs. 81.7\%) (6), confirming it as a new standard of care for such patients.

The rectum is an uncommon primary site for GIST development, representing $<6 \%$ of the cases in the largest reported series (2). Rectal tumors pose several unique challenges to optimal management. The paucity of space in the pelvis renders optimal oncologic surgery more challenging compared to other bowel surgeries and proximity of these lesions to the anal sphincter also increases the potential morbidity of any radical surgery. In rectal adenocarcinoma, local failure is an important factor of first treatment failure and is associated with significant morbidity, with limited success accrued from salvage procedures. The employment of multi-modality perioperative treatment with chemotherapy and radiotherapy has resulted in $\sim 50 \%$ reduction of local recurrences, even in the era of modern surgery with total mesorectal excision $(7,8)$.

In our experience, the local control in rectal GIST was poor and treatment for this disease subset was associated with high morbidity. The present study aimed to evaluate the clinical characteristics of localized, resected rectal GIST in comparison with GIST of other primary sites, and to determine the clinical outcomes and challenges to management of this uncommon GIST location.

\section{Patients and methods}

Study approval. This study was approved by the Institutional Review Board of the National Cancer Singapore (NCCS). The cases with the diagnosis of GIST seen at NCCS between 2002 and 2011 were identified through the institutional diagnostic records system. Diagnoses of GIST were centrally reviewed. Only patients presenting with localized disease and with no distant metastases, who had undergone surgical resection were included in this study.

Patient characteristics. Clinical data were obtained from our GIST database, patient medical charts and electronic medical records. Tumors were classified according to the site of origin (stomach, small intestine, colon and rectum). The type and intent of the surgery was determined for each case based on the medical charts and operation reports. The surgical margins of primary resection were defined as follows: R0, microscopically-negative margins; R1, microscopically-positive margins and R2, gross residual disease. Tumor pathologic characteristics, including size and mitotic rate, were recorded. Details of adjuvant or neo-adjuvant treatment were captured. At the time of relapse, data obtained included nature of relapse (local vs. distant). The cut-off date for reporting data was 1 July, 2011. Survival data were determined based on medical records and cross-referenced against the Singapore Death Registry for patients still living in Singapore.

Statistical analysis. Comparison of the categorical characteristics by primary sites were performed using either the Chi-square test or Fisher's exact test, as appropriate. The median age at the diagnosis of rectal GIST was compared against small intestinal/gastric GIST, using the Mann-Whitney U test.
The overall survival (OS) duration was calculated from the date of diagnosis to the date of death from any cause. Relapse-free survival (RFS) duration was calculated from the date of diagnosis to the date of the first relapse or death. Patients who did not develop any of these time-to-event endpoints were censored at the date of the last follow up. The Kaplan-Meier method was used to estimate survival distribution, while the log-rank test was used to examine the differences between survival curves. The tests were two-sided, and $\mathrm{P}<0.05$ was considered to indicate a statistically significant difference. The analyses were performed using the SAS ${ }^{\circledR} 9.2$ software (SAS Institute, Inc., Cary, NC, USA).

\section{Results}

Clinical characteristics. One hundred and twelve consecutive patients with resected localized GIST were identified based on our records. Nine patients $(8 \%)$ had rectal GIST, whereas the remaining patients had GIST of the stomach $(n=63 ; 56 \%)$, small intestine $(n=37 ; 33 \%)$ and colon $(n=3 ; 3 \%)$. Median follow up was 46 months for the entire cohort and 59 months for patients with rectal GIST. Due to the small patient numbers, patients with colonic GIST were omitted from further analyses.

The median age of patients with rectal GIST was 58 years (range, 37-69 years; 78\% male). These values were not significantly different from patients with GIST of small intestinal or gastric origin. Of the patients with complete pathological data, none with rectal GIST had tumors $>10 \mathrm{~cm}$, compared to $40 \%$ of patients with small intestinal/gastric GIST $(\mathrm{P}=0.044)$. There were no significant differences in the mitotic activity or the eventual risk classification by the original NIH consensus criteria in rectal and small intestinal/gastric GIST. More than $60 \%$ of patients in each anatomic group were classified as high risk (Table I).

Local control and morbidity. The 9 patients with localized rectal GIST underwent curative surgery, of whom $67 \%$ received $\mathrm{R} 0$ resection. This compares unfavorably with GIST patients of small intestinal and gastric origin, of whom $92 \%$ of evaluable patients received surgical resection with microscopically-negative margins $(\mathrm{P}=0.054)$. Notably, of the patients with rectal GIST, peri-operative morbidity was substantial. Three patients required an abdomino-perineal resection (APR). Of the remaining 6 patients treated with resection and bowel anastomosis, 2 patients experienced peri-operative tumor rupture, and 1 patient developed anastomotic wound breakdown and abscess formation. Although not statistically significant, 22\% of rectal GIST patients experienced tumor rupture vs. only $5 \%$ of evaluable patients with small intestinal/gastric GIST $(\mathrm{P}=0.106)$ (Table II).

Only 2 patients with rectal GIST (22\%) received adjuvant imatinib, a proportion similar to that observed in patients with small intestinal and gastric GIST (16 and 30\%, respectively; $\mathrm{P}=0.291)$. The relapse rates for rectal, small intestinal and gastric GIST were 67,51 and $38 \%$ respectively $(\mathrm{P}=0.174)$. Five out of $6(83 \%)$ rectal GIST patients whose disease relapsed experienced local recurrence as a site of the first relapse (either local recurrence only or concomitant local and distant failure). In comparison, only $21 \%$ of first relapses in small intestinal/gastric GIST comprised local failures $(\mathrm{P}=0.005)$. 
Table I. Clinical characteristics and distribution of risk factors.

\begin{tabular}{|c|c|c|c|}
\hline Clinical characteristics & $\begin{array}{l}\text { Rectum } \\
(n=9)\end{array}$ & $\begin{array}{l}\text { Small intestinal/gastric } \\
\qquad(\mathrm{n}=100)\end{array}$ & P-value \\
\hline Median age at diagnosis, years (range) & $58(37-69)$ & $60(17-88)$ & 0.422 \\
\hline \multicolumn{4}{|l|}{ Gender, no. (\%) } \\
\hline Female & $2(22)$ & $38(38)$ & \multirow[t]{2}{*}{0.481} \\
\hline Male & $7(78)$ & $62(62)$ & \\
\hline \multicolumn{4}{|l|}{ Tumor size, no. $(\%)^{\mathrm{a}}$} \\
\hline$\leq 2 \mathrm{~cm}$ & 0 & $2(2)$ & \multirow[t]{4}{*}{0.044} \\
\hline$>2-\leq 5 \mathrm{~cm}$ & $2(22)$ & $18(19)$ & \\
\hline$>5-\leq 10 \mathrm{~cm}$ & $7(78)$ & $38(39)$ & \\
\hline$>10 \mathrm{~cm}$ & 0 & $39(40)$ & \\
\hline \multicolumn{4}{|l|}{ No. of mitoses per $50 \mathrm{HPF}$, no. $(\%)^{\mathrm{a}}$} \\
\hline $0-5$ & $2(22)$ & $32(33)$ & \multirow[t]{3}{*}{0.422} \\
\hline $6-10$ & 0 & $14(14)$ & \\
\hline$>10$ & $7(78)$ & $51(53)$ & \\
\hline \multicolumn{4}{|l|}{$\mathrm{NIH}$ risk classification, no. $(\%)^{\mathrm{a}}$} \\
\hline Very low/low & 0 & $11(11)$ & \multirow[t]{3}{*}{0.618} \\
\hline Intermediate & $2(22)$ & $16(17)$ & \\
\hline High & $7(78)$ & $70(72)$ & \\
\hline
\end{tabular}

${ }^{\text {a} B a s e d ~ o n ~ c o m p l e t e ~ p a t h o l o g i c a l ~ d a t a ~ f o r ~} 106$ patients (9 rectal, 97 small intestinal/gastric). HPF, high-power field; NIH, National Institutes of Health.

Table II. Local control and morbidity.

\begin{tabular}{lccc}
\hline & $\begin{array}{c}\text { Rectum } \\
(\mathrm{n}=9)\end{array}$ & $\begin{array}{c}\text { Small intestinal/ } \\
\text { gastric (n=100) }\end{array}$ & P-value \\
\hline $\begin{array}{l}\text { Resection margins, } \\
\text { no. }(\%)^{\mathrm{a}}\end{array}$ & & & \\
Negative & $6(67)$ & $86(91)$ & 0.054 \\
Positive & $3(33)$ & $8(9)$ & \\
$\begin{array}{l}\text { Tumor rupture, } \\
\text { no. }(\%)^{\mathrm{b}}\end{array}$ & & & \\
No & $7(78)$ & $93(95)$ & 0.106 \\
Yes & $2(22)$ & $5(5)$ & \\
\hline
\end{tabular}

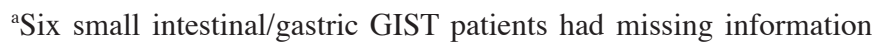
on resection margins. ${ }^{b}$ Two small intestinal/gastric GIST patients had missing information on tumor rupture.

Relapse-free and overall survival. Median OS for the entire study cohort was 141 months, with no statistically significant differences between the cohorts of patients with rectal (162 months), small intestinal (not reached) and gastric GIST (141 months) $(\mathrm{P}=0.363)$. Median RFS for the entire cohort was 51 months, with no statistically significant difference noted between rectal (54 months), small intestinal (36 months) and gastric GIST (56 months) $(\mathrm{P}=0.468)$, as shown in Figs. 1 and 2 .

\section{Discussion}

In this study, the rectum was confirmed to be an uncommon primary site for GIST, representing only $8 \%$ of the patients with resected localized GIST. In comparison with patients with small intestinal and gastric GIST, there does not seem to be any unique pattern of gender or age at presentation in rectal GIST. Notably, rectal tumors in our series were significantly smaller compared to small intestinal/gastric tumors. This could possibly be correlated with the propensity for rectal masses to become symptomatic earlier than tumors in the upper gastrointestinal tract. The anatomic groups did not differ in terms of the other major prognostic factor, mitotic rate, possibly accounting for the lack of differences in NIH criteria risk categories in the groups.

In this study, the number of patients with resected localized GIST, who received adjuvant imatinib, was relatively low ( $<30 \%$ in each anatomic group), in spite of the majority of patients ( $<60 \%$ in each anatomic group) being at high risk. This is likely due to the fact that imatinib was only recently approved for use in this indication. In the landmark adjuvant study conducted by the ACOSOG group prior to the SSGXVIII study, the absence of OS benefit was likely to be another contributory factor (5). Comparing patients regarding primary GIST locations, no statistically significant difference was observed in adjuvant imatinib use in rectal GIST and small intestinal/gastric GIST patients.

Notably, although the overall relapse rates for patients with rectal GIST was not significantly different from those with small intestinal/gastric GIST, the patterns of relapse differed significantly. Of the patients whose disease recurred, 
A

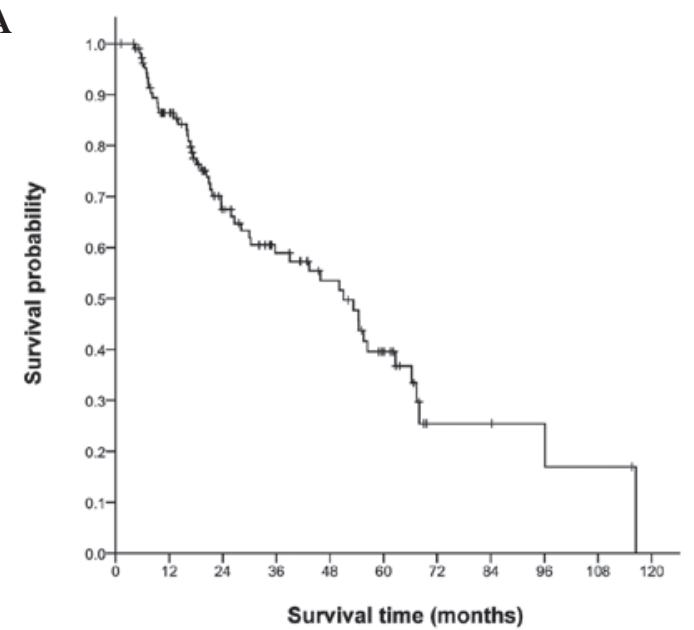

B

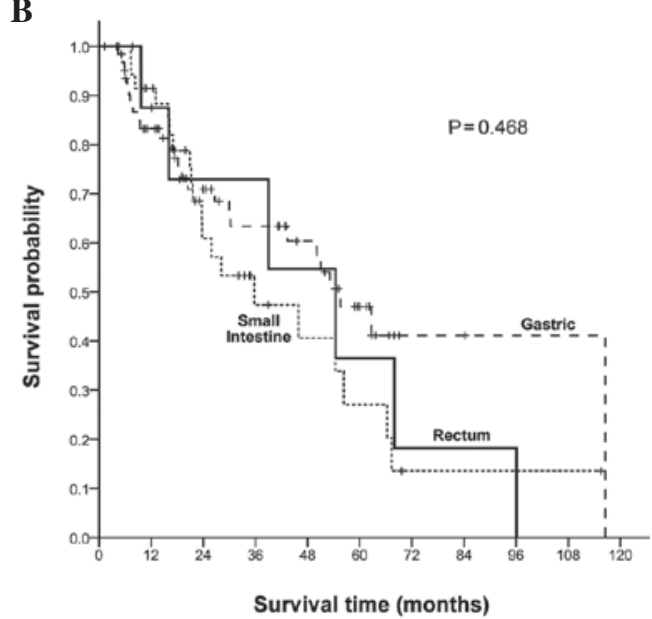

Figure 1. Relapse-free survival of (A) all the patients and (B) based on anatomic site.

A

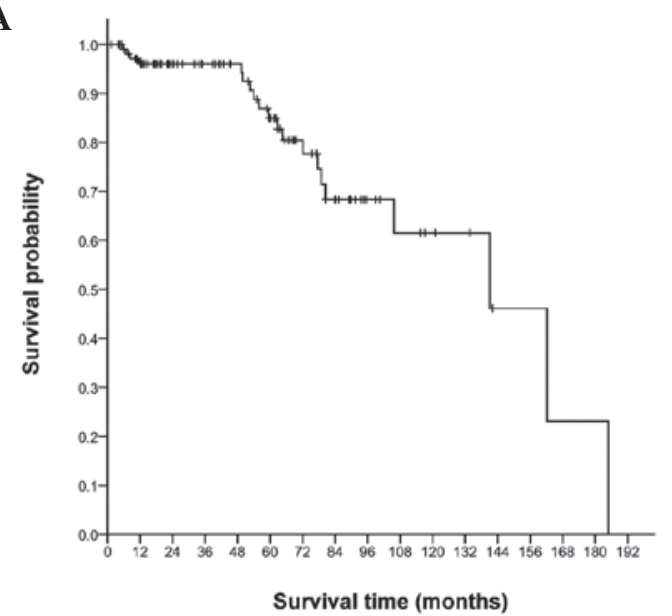

B

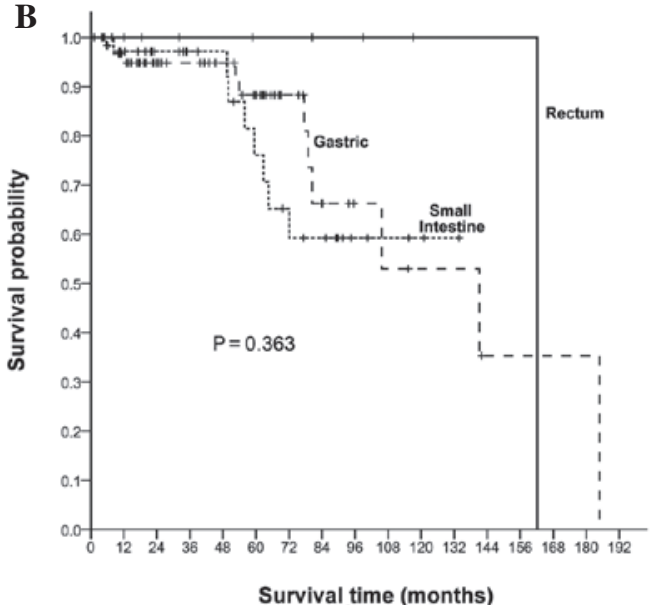

Figure 2. Overall survival of (A) all the patients and (B) based on anatomic site.

$83 \%$ with rectal GIST experienced local failure at the first relapse, compared to $21 \%$ of patients with small intestinal and gastric GIST. This finding is probably due to the lower rates of R0 surgical resection for rectal GIST, compared to small intestinal and gastric GIST, a difference of almost statistically significant difference $(\mathrm{P}=0.054)$. The significant challenges of complete surgical extirpation in the tight confines of the pelvis have likely contributed to this result. This difficulty with an optimal local control is the most noteworthy in light of the fact that the rectal tumors in this series were significantly smaller compared to the non-rectal (small intestinal/gastric) tumors. The prominence of local failure in rectal GIST has been observed by several groups. In a review by Peralta (9) the local recurrence rate was 75\% in tumors $>5 \mathrm{~cm}$ (regardless of the mitotic rate), and $62 \%$ in tumors $<5 \mathrm{~cm}$ (with $>5$ mitoses per $50 \mathrm{HPF}$ ). The failure of the local disease control has been shown to lead to poor overall outcomes, even after secondary surgery (10). In addition, the morbidity associated with the local resection of rectal GIST was clearly substantial, with 3 patients needing sphincter-compromising APRs, 2 experiencing peri-operative tumor rupture and 1 suffering from an anastomotic breakdown/abscess formation. The proportion of patients with rectal GIST experiencing tumor rupture was $>4$-fold of the non-rectal GIST patients ( 22 vs. 5\%; $\mathrm{P}=0.106$ ). Although this numerical difference did not reach statistical significance, it might be due to the small sample size of our study population. These findings are notable, given that the non-rectal tumors were significantly larger compared to the rectal tumors, underlining the prominent local morbidity associated with rectal GIST. While no formal quality of life measures were recorded, the high rates of local failure and the significant number of events associated with peri-operative morbidity had a negative impact on outcomes of patients with resected rectal GIST. In addition to an increasing morbidity, tumor rupture has been evaluated as an independent adverse prognostic factor by Rutkowski et al (11), for which patients would require 3 years of adjuvant imatinib with its attendant toxicities and cost, based upon the data from the recent SSGXVIII study (6).

Thus, the significant local morbidity and high rates of local failure associated with the surgical treatment of rectal GIST may signal the need for novel, multi-modal therapeutic strategies to optimize outcomes. Multi-modality peri-operative therapy has been shown to improve outcomes and is currently 
the mainstay of treatment in rectal adenocarcinoma $(7,8)$, a disease sharing the anatomical constraints and difficulties with optimal local control seen in rectal GIST. Neo-adjuvant imatinib has been shown to be safe and well-tolerated in the treatment of locally advanced GIST. The median time to best response with imatinib in advanced GIST has been reported to be between 3 and 4 months (12). In their study, Blesius et al (13) demonstrated that up to $36 \%$ of the patients with locally advanced non-metastatic GIST deemed upfront unresectable received neo-adjuvant imatinib and subsequently underwent surgery by the local surgeons, who had initially advised against operation. Neo-adjuvant imatinib has also been shown to facilitate anal sphincter preservation without compromising optimal local control in patients with rectal GIST initially deemed to necessitate APR (14). These data argue strongly for neo-adjuvant imatinib in rectal GIST, especially in patients deemed unresectable or for whom APR is being considered. Upfront surgical resection resulting in positive margins and/or peri-operative tumor events is likely to exacerbate morbidity and contribute to increased recurrences and sub-optimal outcomes.

Although the use of radiation is not traditionally associated with the curative treatment of GIST, its role requires further examination, particularly when combined with imatinib. Preclinical data suggest imatinib to be a radiation-sensitizer (15), while isolated reports have suggested the potential efficacy of radiation in managing rectal GIST when combined with imatinib (16).

Thus, rectal GIST is a rare subset of GIST, for which data remain scant and outcomes are sub-optimal with surgical resection alone. In this study, in spite of being significantly smaller compared to GIST of other common sites, rectal GIST was demonstrated to be associated with significantly higher rates of positive surgical margins and local relapses attributable to the unique anatomical location of the primary tumor. The morbidity of upfront rectal surgery in this subset of GIST is also substantial.

Increased local relapse and high peri-operative morbidity render the evaluation of fresh approaches necessary to improve outcomes in this disease. A multi-modality approach incorporating neo-adjuvant and/or adjuvant imatinib in the appropriate patient setting should be strongly considered. The involvement of peri-operative radiation in rectal GIST also deserves further clinical evaluation in a controlled, prospective clinical trial setting.

\section{References}

1. Fletcher CD, Berman JJ, Corless C, et al: Diagnosis of gastrointestinal stromal tumors: a consensus approach. Hum Pathol 33: 459-465, 2002.

2. Miettinen M and Lasota J: Gastrointestinal stromal tumors: pathology and prognosis at different sites. Semin Diagn Pathol 23: 70-83, 2006.

3. Joensuu H: Risk stratification of patients diagnosed with gastrointestinal stromal tumor. Hum Pathol 39: 1411-1419, 2008.

4. Rutkowski P, Nowecki ZI, Michej W, et al: Risk criteria and prognostic factors for predicting recurrences after resection of primary gastrointestinal stromal tumor. Ann Surg Oncol 14: 2018-2027, 2007.

5. Dematteo RP, Ballman KV, Antonescu CR, et al: Adjuvant imatinib mesylate after resection of localised, primary gastrointestinal stromal tumour: a randomised, double-blind, placebo-controlled trial. Lancet 373: 9669-1097, 2009.

6. Joensuu H, Eriksson M, Hatrmann J, et al: Twelve versus 36 months of adjuvant imatinib as treatment of operable GIST with a high risk of recurrence: final results of a randomized trial (SSGXVIII/AIO) (abs. LBA1). J Clin Oncol 29: 2011.

7. Van Gijn W, Marijnen CA, Nagtegaal ID, et al: Preoperative radiotherapy combined with total mesorectal excision for resectable rectal cancer: 12-year follow-up of the multicentre, randomised controlled TME trial. Lancet Oncol 12: 575-582, 2011.

8. Sauer R, Becker H, Hohenberger W, et al: Preoperative versus postoperative chemoradiotherapy for rectal cancer. N Engl J Med 351: 1731-1740, 2004.

9. Peralta EA: Rare anorectal neoplasms: gastrointestinal stromal tumor, carcinoid, and lymphoma. Clin Colon Rectal Surg 22: 107-114, 2009.

10. Dong C, Jun-Hui C, Xiao-Jun Y, Mei K, Bo W, Chen-Fe J and Wei-Li Y: Gastrointestinal stromal tumors of the rectum: clinical, pathologic, immunohistochemical characteristics and prognostic analysis. Scand J Gastroenterol 42: 1221-1229, 2007.

11. Rutkowski P, Bylina E, Wozniak A, et al: Validation of the Joensuu risk criteria for primary resectable gastrointestinal stromal tumour-the impact of tumour rupture on patient outcomes. Eur J Surg Oncol 37: 890-896, 2011.

12. Verweij J, Casali PG,Zalcberg J, et al: Progression-free survival in gastrointestinal stromal tumours with high-dose imatinib: randomised trial. Lancet 364: 1127-1134, 2004.

13. Blesius A, Cassier PA, Bertucci F, et al: Neoadjuvant imatinib in patients with locally advanced non metastatic GIST in the prospective BFR14 trial. BMC Cancer 15: 72, 2011.

14. Wang JP, Wang T, Huang MJ, Wang L, Kang L and Wu XJ: The role of neoadjuvant imatinib mesylate therapy in sphincter-preserving procedures for anorectal gastrointestinal stromal tumor. Am J Clin Oncol 34: 314-316, 2011.

15. Choudhury A, Zhao H, Jalali F, et al: Targeting homologous recombination using imatinib results in enhanced tumor cell chemosensitivity and radiosensitivity. Mol Cancer Ther 8: 203-213, 2009.

16. Ciresa M, D'Angelillo RM, Ramella S, et al: Molecularly targeted therapy and radiotherapy in the management of localized gastrointestinal stromal tumor (GIST) of the rectum: a case report. Tumori 95: 236-239, 2009. 